\title{
The Targeted Antitumor Effects of C- PC/CMC-CD59sp Nanoparticles on HeLa Cells in Vitro and in Vivo
}

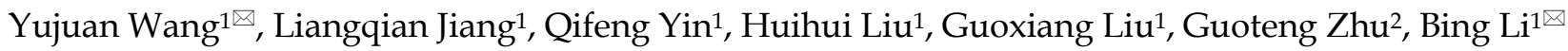 \\ 1. Department of genetics and cell biology, Basic medical college, 308 Ningxia Road, Qingdao University, Qingdao, China, 266071. \\ 2. Affiliated Hospital of Qingdao University, Qingdao, China, 266000.
}

$\triangle$ Corresponding authors: Bing Li, Department of genetics and cell biology, Basic medical college, 308 Ningxia Road, Qingdao University, Qingdao, China, 266071. Tel: 86-532-83780060; Email: libing_516@qdu.edu.cn, and Yujuan Wang, Email: lduwangyujuan@163.com

(c) Ivyspring International Publisher. This is an open access article distributed under the terms of the Creative Commons Attribution (CC BY-NC) license (https://creativecommons.org/licenses/by-nc/4.0/). See http://ivyspring.com/terms for full terms and conditions.

Received: 2017.05.17; Accepted: 2017.06.30; Published: 2017.08.25

\begin{abstract}
The novel C-PC/CMC-CD59sp-NPs were made by carbocymethyl chitosan (CMC) loading C-phycocyanin (C-PC) with the lead of CD59 specific ligand peptide (CD59sp) for targeting, and the characteristics and targeted anti-tumor mechanism were explored in order to realize the targeted therapy of C-PC on the growth of HeLa cells both in vitro and vivo. The targeting nanoparticles were synthesized by ionic-gelation method, and the optimal condition was selected out by orthogonal analysis. The properties of nanoparticles were observed by laser particle analyzer and dynamic light scattering (DLS) and Fourier Transform Infrared Spectrometer (FTIR). The effects of nanoparticles on the proliferation of HeLa cells in vitro were assessed by MTT assay. The mice model with tumor was constructed by subcutaneous injection of HeLa cells into the left axilla of NU/NU mice. The weight of tumor and the spleen were tested. The expression quantities of cleaved caspase-3, Bcl-2 were determined by western blot and immunofluorescent staining. Results showed the morphology of the finally prepared nanoparticles was well distributed with a diameter distribution of $200 \pm 11.3 \mathrm{~nm}$ and zeta potential of $-19.5 \pm 4.12 \mathrm{mV}$. Under the guidance of CD59sp, the targeting nanoparticles could targetedly and efficiently arrive at the surface of HeLa cells, and had obvious inhibitory effect on HeLa cells proliferation both in vitro and vivo. Moreover, the nanoparticles could induce cell apoptosis by up-regulation of cleaved caspase-3 proteins expression, but down-regulation of $\mathrm{Bcl}-2$ and cyclinDl proteins. Our study provided a new idea for the research and development of marine drugs, and supplied a theoretical support for the target therapy of anticancer drug.
\end{abstract}

Key words: C-phycocyanin; targeting nanoparticles; carboxymethyl chitosan; CD59- specific ligand peptide; HeLa cells

\section{Introduction}

Tumor targeted drug delivery system has become an important part of cancer treatment today. Due to its characteristics such as specificity and selective drug delivery, many shortcomings of traditional drug delivery can be overcomed. The side effects of drugs are reduced, biologic half-life of the drugs in the body is extended and the effective functions of drugs are enhanced[1].

Spirulina platensis has a large number of biological active substances such as C-phycocyanin, $\beta$-carotene and polysaccharides, so it is known as "the 21st century's best health care products" by Food and
Agriculture Organization[2]. C-phycocyanin (abbreviated as C-PC) is a natural photosynthetic pigment and bright blue, composed of rich amino acids especially essential amino acids. C-phycocyanin is proved to have plenty of effects such as anti-aging, oxidation resistance, radiation resistance, fatigue resistance and anti-diabetes. Moreover, C-phycocyanin is non-toxic, safe, good water soluble and strong staining, so it is widely used in food, cosmetics, dyes and other industries[3]. C-phycocyanin has red fluorescent and can be used as a fluorescent marker[4]. Bing $\operatorname{Li}[5,6]$ found that 
C-phycocyanin can enhance the human immunity and inhibit tumor growth, but because of its poor stability and susceptibility to protease degradation, so the application in the pharmaceutical industry was greatly restricted. Therefore, how to design a safe and stable drug (C-phycocyanin) delivery system is a key problem currently.

The rise of nanomedicine, makes it possible to transfer the stable proteins. Chitosan is formed by deacetylation of chitin and has many characteristics of the natural polymer materials such as non-toxity, biocompatibility, biodegradability and so on[7]. Chitosan has medical efficacy including antisepsis and anti-inflammation, antitumor and promoting wound healing, but its insolubility in water greatly limits its application. Carboxymethyl chitosan (CMC) is constructed by carboxylation modification of chitosan, and because of its good water solubility and biocompatibility, non-toxic, antibacterial characteristics, CMC has been applied in many industries and researches, especially in drug carrier. Previous research found that many natural medicine or chemotherapy drugs were packaged in CMC to realize slow-release, improve efficacy, reduce less side effects and inhibit tumor growth[8]. We chose carboxymethyl chitosan as a carrier to embed C-phycocyanin by using the ionic crosslinking method $\left(\mathrm{CaCl}_{2}\right.$ acted as crosslinking agent) to prepare C-PC/CMC, which not only improved the stability of the C-PC, but also realized the slow release of C-PC. Our study provided a new basis for C-PC as a potential anti-cancer drug.

In order to improve the target ability of drugs, on the basis of the difference between tumor cells and normal cells, the size of the nanoparticles and the sensitivity to $\mathrm{pH}$ and temperature were controlled to realize passive targeting drug delivery [9-11]. In addition, some molecules were found in tumors with high expression such as CD40, EGFR LDLr [12, 13] which help to realize the active targeting drug delivery. Research found that CD59 gene were highly expressed in many solid tumors, such as colon cancer, cervical cancer, ovarian cancer and prostate cancer cells, but less expressed in normal cells[14]. CD59 is a kind of membrane complement regulatory protein, and its high expression will inhibit the formation of complement attack complex (MAC) and cause the immune escape of tumor cells. Our previous study [15] screened out CD59 specific ligand peptide (CD59sp) which could effectively bind to CD59 molecules by phage peptide library. CD59sp-CD59 complex formation resulted in the targeting delivery of special drugs into tumor cells.

Preliminary studies had proved C-phycocyanin, carboxymethyl chitosan and CD59 specific ligand peptide have varying degrees of anti-tumor effect. Our study took advantage of nanomedicine, and C-PC, CMC and CD59sp were combined to construct C-PC/CMC-CD59sp NPs to take anti-cancer effect. $\mathrm{CMC}$ acted as a carrier to enclose the C-PC to form C-PC/CMC nanoparticles, CD59sp acted as a guide, and with the guidance of CD59sp, the targeted C-PC/CMC-CD59sp NPs were synthesized. The targeted delivery of nanoparticles were achieved, furthermore, the targeting, security and the anti-tumor effect of the targeted nanoparticles were detailedly explored.

\section{Materials and Methods}

\section{Materials}

Carboxymethyl chitosan (MW 6-10 kDa; deacetylation degree $\geq 95 \%$ and degree of substitution $\geq 90 \%$ ) was purchased from Qingdao Honghai Bio-Tech Co. Ltd., Qingdao, China. C-phycocyanin was purchased from Taizhou Binmei Biotechnology Co., Ltd., Taozhou, China. CD59-specific ligand peptide was synthesis by Chinese Peptide Company, Hangzhou, China. HeLa cells were provided by the Affiliated Hospital of Qingdao University. Methyl thiazolyl tetrazolium (MTT) was purchased from Beijing Solarbio Science \& Technology, Beijing, China. Rabbit antihuman Bcl-2/ cyclinD1 monoclonal antibodies were purchased from Abcam Company. Rabbit antihuman caspase- 3 and cleaved caspase- 3 monoclonal antibodies were purchased from Proteintech Group, Inc. Immunofluorescence staining kit was purchased from Boster Biological Engineering Co., Ltd. All other used chemicals reached analytical grade.

\section{Synthesis of C-PC/CMC NPs}

The C-PC/CMC NPs were made by the ionic-gelation method. Briefly, $\mathrm{CaCl}_{2}$ was added dropwise into $\mathrm{CMC}$ solution that was pre-incubated with C-phycocyanin, until the solution became turbid. Nanoparticles were spontaneously obtained under constant stirring at $4^{\circ} \mathrm{C}$, in the dark, for $30 \mathrm{~min}$. The prepared NPs were separated by centrifugation at $9000 \times \mathrm{g}$ for $15 \mathrm{~min}$ at $4^{\circ} \mathrm{C}$, and then sterilized using a syringe filter with pore size of $0.22 \mu \mathrm{m}$.

\section{Synthesis of C-PC/CMC-CD59sp NPs}

CD59sp was coupled with C-PC/CMC NPs by amine coupling, using EDC-activated carboxyl group conjugation chemistry. In this procedure, about $2 \mathrm{mg}$ of EDC was added to $5 \mathrm{mg}$ of C-PC/CMCNP samples in PBS. The $\mathrm{pH}$ of the solution was adjusted to 5.6, with stirring (600rpm) at room temperature for $1 \mathrm{~h}$. Then, $1 \mathrm{mg} / \mathrm{mL}$ of CD59sp was added to the solution and stirred overnight at $4^{\circ} \mathrm{C}$ in the dark to make 
C-PC/CMC-CD59sp NPs. NPs were subsequently separated by centrifugation $(9000 \times \mathrm{g})$ for $15 \mathrm{~min}$. The pellets were collected and used for further characterization and studies.

\section{Evaluation of NPs by laser particle analyzer}

Zeta potential is commonly used to characterize the surface charge property of nanoparticles[16]. Size and zeta potential of nanoparticles were determined using Malvern NanoZS (Malvern, Zeta, Worcestershire, UK). A tenfold dilution of the sample in pure water in a total volume of $1 \mathrm{~mL}$ was subjected to a particle size analyzer at $25^{\circ} \mathrm{C}$.

\section{Infrared spectrometer detection}

The dry nanoparticles were mixed with $\mathrm{KBr}$ powder and then were grinded to powder, and subsequently detected by the infrared spectrometer and scanning range was $500-4000 \mathrm{~cm}^{-1}$.

\section{The experimental groups}

HeLa cells were divided into four groups: (1) a control group, not treated with any drug; (2) the C-PC group; (3) the untargeted group, treated with C-PC/CMC NPs; and (4) the targeted group, treated with C-PC/CMC- CD59sp NPs. Experimental drug concentration was half of IC50 value, and the drugs and medium were mixed at the volume ratio of 1:1.

\section{MTT assay}

The cells were divided into positive control group (no treatment) and negative control group (1\% (v/v) Triton X-100). $100 \mu$ l HeLa cells ( $1 \times 10^{7} /$ well) at the log phase were incubated in 96-well culture plate overnight and treated with $100 \mu \mathrm{l}$ different concentrations of drugs $(1,10,50,100,200 \mu \mathrm{g} / \mathrm{ml})$ for $48 \mathrm{~h}$. Each group had five parallel wells. $20 \mu \mathrm{l} 5$ $\mathrm{mg} / \mathrm{ml}$ MTT was added to each well for $4 \mathrm{~h}$ followed by $150 \mu \mathrm{l}$ DMSO treatment for $10 \mathrm{~min}$. The optical density at $490 \mathrm{~nm}$ (OD490nm) was detected and the survival rate of each group was calculated. Calculation formula was: the survival rate $(\%)=$ $\mathrm{OD}_{\text {sample }} / \mathrm{OD}_{\text {control. }}$ IC50 value of the each group was calculated.

\section{Cellular morphological changes detected by confocal microscope}

Cells grew on the glass slide overnight. The fresh media were replaced, and the cells were treated with FITC-labeled CMC/C-PC CD59sp nanoparticles. After $24 \mathrm{~h}$, cells were fixed with $4 \%$ paraformaldehyde for $10 \mathrm{~min}$ at room temperature away from light, then rinsed with PBS. Finally, confocal microscope was applied to view the cellular morphology.

\section{Changes of cell cycle by flow cytometry}

For fixed cell analysis, the trypsinized cells were resuspended in ice-cold ethanol/PBS $(70 \% \mathrm{v} / \mathrm{v})$ and kept at $-20^{\circ} \mathrm{C}$ until ready to use. Before flow cytometry analysis, the fixed cells were resuspended in $0.5 \mu \mathrm{M}$ vybrant violet dye or propidium iodide and RNase A solution. Flow analyses were performed on a LSR Fortessa driven by BD's FACS Diva software, version 6.1.3 (Becton Dickinson, San Jose, CA).

\section{In vivo Study: The construction of mice model with tumor and grouping}

A total of 20 adult BALB/c nude mice (females, 20-22g) were used for the study. To establish the grafted tumor model, $5 \times 10^{6}$ cells mixed with Basement Membrane Matrix (BD Biosciences, San Jose, CA, USA) were injected subcutaneously (sc) into the right flank of the mice. Fifteen days after injection, when the tumors attained an average volume of 100 $\mathrm{mm}^{3}$, the 20 nude mice were weighed and randomly divided into four groups: control group, the CMC-CD59sp treated group, the untargeted group (C-PC/CMC NPs treated group), and the targeted group (C-PC/CMC- CD59sp NPs treated group). These agents were injected into the area of tumors and the duration of drug treatment was 20 days. Two days after drug with drawal, the mice were executed, and then tumors and spleens were picked out. The formation of subcutaneous tumours was monitored and measured with a digital calliper. The tumour volumes were calculated based on the formula $\mathrm{LW}^{2}$ $/ 2$, where $\mathrm{L}$ is the length and $\mathrm{W}$ is the shortest width of the tumour. All studies were approved by the Qingdao University institutional animal care and use committee. Animals were housed under standard conditions with ad libitum food and water and a 12:12 light: dark cycle at the Qingdao University facilities.

\section{Bcl-2/Cleaved Caspase-3 proteins expression determined by immunofluorescence}

The slices were conventionally dewaxed, immersed in $0.01 \mathrm{M}$ citrate solution, and then heated to boiling status in a microwave oven. After washed with PBS for three times, the slides were blocked with normal goat serum at a 1:10 dilution for $10 \mathrm{~min}$ and then incubated in the rabbit anti-human Bcl-2/cleaved caspase-3 monoclonal antibody for $2 \mathrm{~h}$. The cells were then washed with PBS and incubated in the goat anti-rabbit secondary immunoglobulin for $30 \mathrm{~min}$ at room temperature. After being washed with PBS for three times, the cells were stained with SABC-Cy3 at a 1:100 dilution for $30 \mathrm{~min}$ in dark. The slides were observed under a fluorescence microscope. 


\section{Western bolt}

Tumor tissues were homogenized and lysed with lysis buffer. The protein concentrations were determined using the BCA protein assay reagent (Solarbio, Beijing, China). For western blot analysis, equal amounts of total proteins were mixed with SDS sample buffer and separated by SDS-polyacrylamide gel electrophoresis. After electrophoresis, protein was transferred onto a PVDF membrane (Millipore, Billerica, MA, USA) and blocked for $1 \mathrm{~h}$ in $5 \%$ BSA. The membrane was incubated with primary antibodies at $4^{\circ} \mathrm{C}$ overnight and Horseradish peroxidase- (HRP-) conjugated anti-rabbit IgG secondary antibody for $1 \mathrm{~h}$. Protein bands were visualized using enhanced chemiluminescence reagents according to the manufacturer's instructions. The densities of protein bands were determined by Image J software. The proteins levels were normalized with respect to the $\beta$-actin level, and the grayscale ratio of protein $/ \beta$-actin was calculated.

\section{Statistics analysis}

The experiments were carried out in triplicate and values were shown as the mean \pm standard deviation (SD). Differences among multiple groups were analyzed by two-way ANOVA using SigmaStat software. The mean values for biochemical data from two groups were compared by 2-tailed Student's t-test. All statistical tests with $\mathrm{P}<0.05$ were considered significant. SPSS 12.0 software was used for statistical analysis.

\section{Results}

\section{Particle size distribution}

The C-PC/CMC nanoparticles detected by laser particle analyzer were about $200 \pm 11.3 \mathrm{~nm}$ (Figure 1A). The zeta potential of CMC alone was found to be in a range of -45 to $-36 \mathrm{mV}$ (Figure 1B). For C-PC alone, the surface charge was determined to be in a range of -23 to $-14 \mathrm{mV}$ (Figure 1C). A zeta potential in the range of -24 to- $16 \mathrm{mV}$ was used for the selection of CMC/C-PC NPs which were made by the mixtion ratio of CMC: C-PC (w/w) was $3: 1$, with $2 \mathrm{mg} / \mathrm{mL} \mathrm{CMC} \mathrm{and} 1$ $\mathrm{mg} / \mathrm{mL} \quad \mathrm{CaCl}_{2}$ (Figure 1D). The synthesized C-PC/CMC nanoparticles were negatively charged but compared with the blank chitosan, negative charges were reduced.

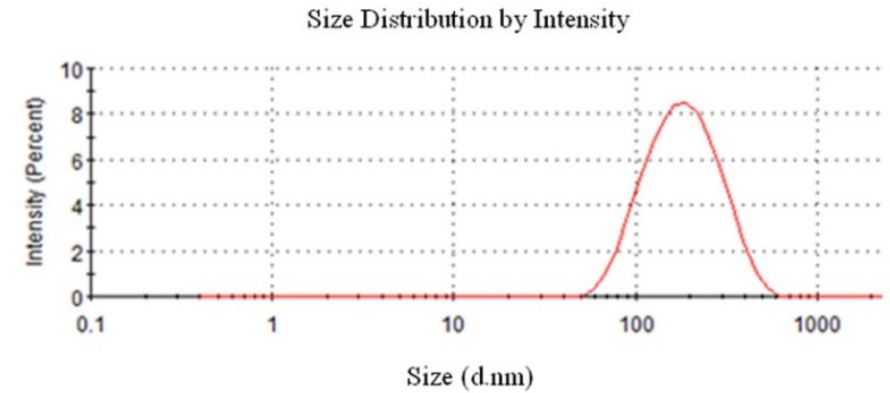

B

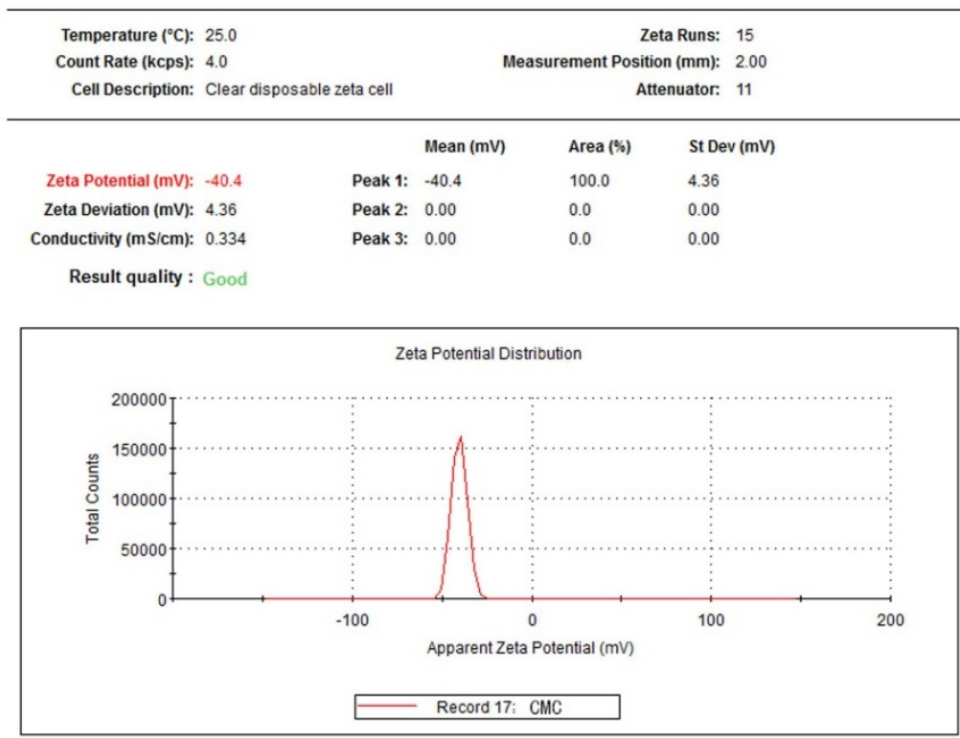


C

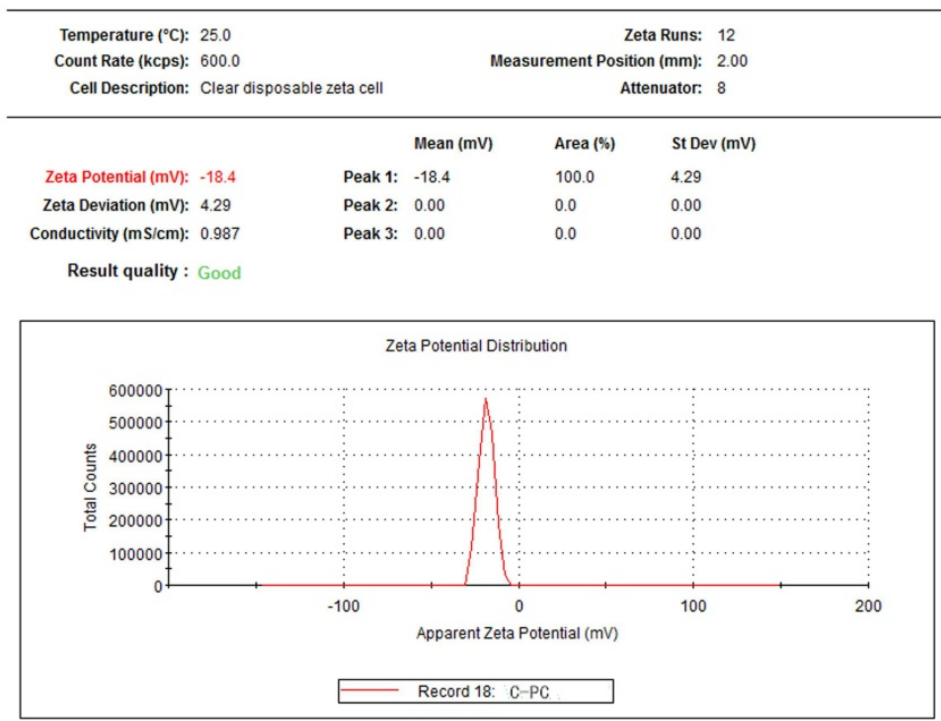

D

\begin{tabular}{|c|c|c|c|c|c|}
\hline \multirow{4}{*}{$\begin{array}{r}\text { Temperature }\left({ }^{\circ} \mathrm{C}\right): \\
\text { Count Rate }(\mathrm{kcps}): \\
\text { Cell Description: }\end{array}$} & \multicolumn{2}{|l|}{25.0} & \multicolumn{3}{|c|}{ Zeta Runs: 12} \\
\hline & \multicolumn{2}{|l|}{122.3} & \multicolumn{3}{|c|}{ Measurement Position $(\mathrm{mm}): \quad 2.00$} \\
\hline & Clearo & zeta cell & \multicolumn{3}{|c|}{ Attenuator: 9} \\
\hline & & & Mean $(\mathrm{mV})$ & Area (\%) & St Dev (mV) \\
\hline Zeta Potential (mV): & -19.5 & Peak 1: & -19.5 & 100.0 & 4.12 \\
\hline Zeta Deviation $(\mathrm{mV})$ : & 4.12 & Peak 2: & 0.00 & 0.0 & 0.00 \\
\hline Conductivity $(\mathrm{mS} / \mathrm{cm})$ : & 0.419 & Peak 3: & 0.00 & 0.0 & 0.00 \\
\hline
\end{tabular}

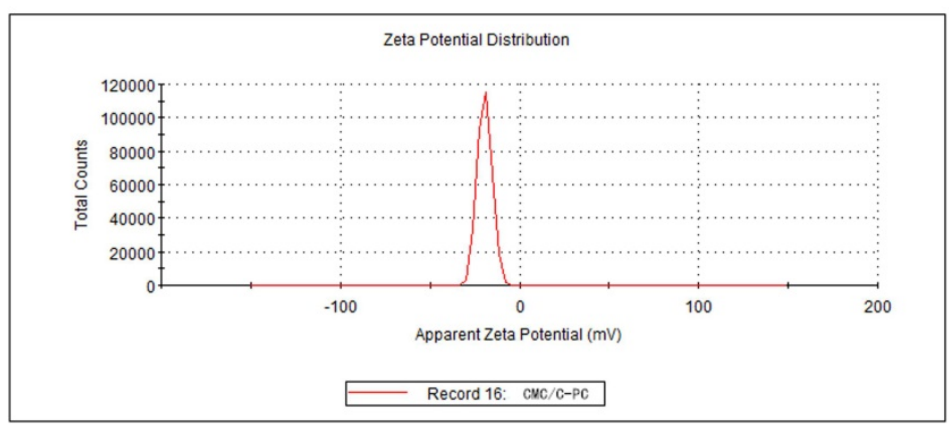

Fig 1. Characterization of nanoparticles. (A) Particle size distribution of C-PC/CMC. The average diameter was $200 \pm 10.5 \mathrm{~nm}$. (B) Zeta potential report of CMC alone with a zeta potential of $-40.4 \pm 4.36 \mathrm{mV}$. (C) For C-PC alone, the surface charge was determined to be $-18.4 \pm 4.29 \mathrm{mV}$. (D) A zeta potential of CMC/C-PC complexes was $-19.5 \pm 4.12 \mathrm{mV}$.

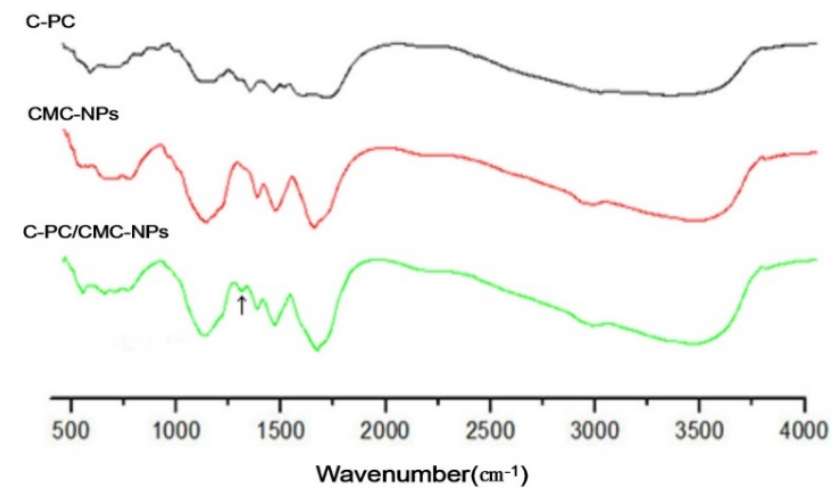

Fig 2. Infrared spectrogram of C-PC, CMC-NPs and C-PC/CMC-NPs. Compared with CMC NPs and C-PC, chemical bonds on the surface of C-PC/CMC NPs was at $1364 \mathrm{~cm}^{-1}$ and hydrogen bonds might form.

\section{Changes of functional groups}

The analytic results of infrared spectrum of CMC NPs and C-PC/CMC NPs were shown in figure 2. Compared with CMC NPs and C-PC, chemical bonds on the surface of C-PC/CMC NPs at $1364 \mathrm{~cm}^{-1}$ changed and hydrogen bonds might form. The results suggested cross linking reaction between CMC and C-PC formed and led to the change of the chemical bonds.

\section{Inhibitory effects of nanoparticles on cell proliferation}

As shown in figure 3, C-PC, C-PC/CMC NPs, C-PC/CMC-CD59sp NPs could inhibit the growth of 
HeLa cells, and the inhibitory rate was positively correlated with drug concentration. The inhibition effect of targeted nanoparticles was significantly higher than the rest of the groups. The half inhibition concentration (IC50 value) of C-PC, C-PC/CMC NPs, C-PC/CMC-CD59sp NPs for HeLa cells was 155.20, 58.08 and $21.46 \mu \mathrm{g} / \mathrm{ml}$, respectively. Therefore, results suggested that the anticancer effects of C - PC embraced in nanoparticles were well reserved and further enhanced in targeted C-PC/CMC-CD59sp nanoparticles.

\section{Intake of nanoparticles into target cells}

Under the guidance of CD59 peptide ligand, through its specific combination of CD59sp with CD59 on cell surface, targeted nanoparticles gathered on the cell surface, and then were engulfed into cells.

At the same time, the intake of drugs into HeLa cells was observed at different time point (Figure 4). The nuclei stained with DAPI staining, showed blue fluorescence, and C-PC illuminated own red fluorescence. With the increase of drug action time, fluorescence intensity was increased. As shown in figure $4 \mathrm{~A}$, after $1 \mathrm{~h}$, fluorescence intensity was significantly less than $4 \mathrm{~h}$ treatment. We also found that $4 \mathrm{~h}$ later, the red fluorescence was not evenly distributed in cells, but gathered in some regions, which suggested the drug was selective but not passive intake, so we realized targeted delivery of C-PC/CMC-CD59sp nanoparticles into HeLa cells, and this was time dependent.

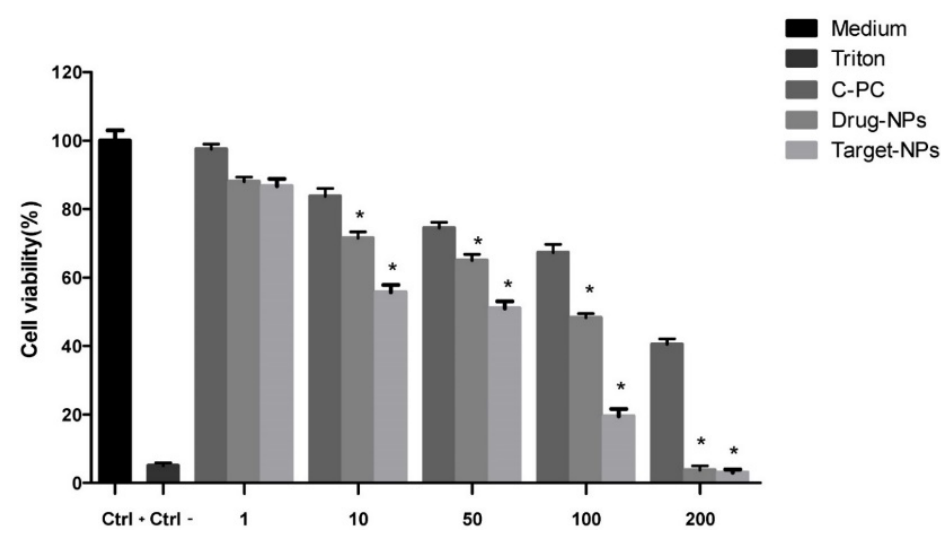

Fig 3. MTT assay. The inhibitory rate of different drug treatments on HeLa cells. Compared with the control group, different drugs have different inhibitory effects on Hela cells. The inhibitory effect of C-PC/CMC-NPs and C-PC/CMC-CD59sp-NPs was higher than that of C-PC. The difference was significant ( $P<0.05)$.

\section{DAPI}

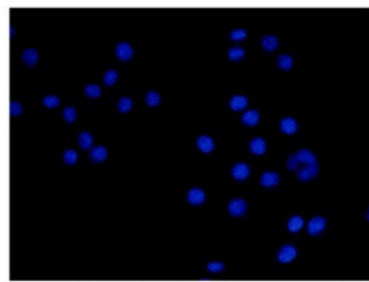

$\mathbf{A}$

B

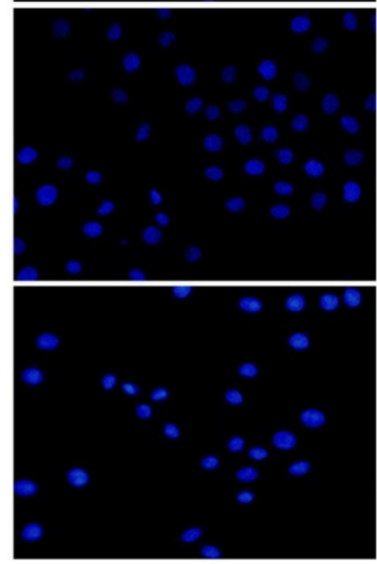

C-PC
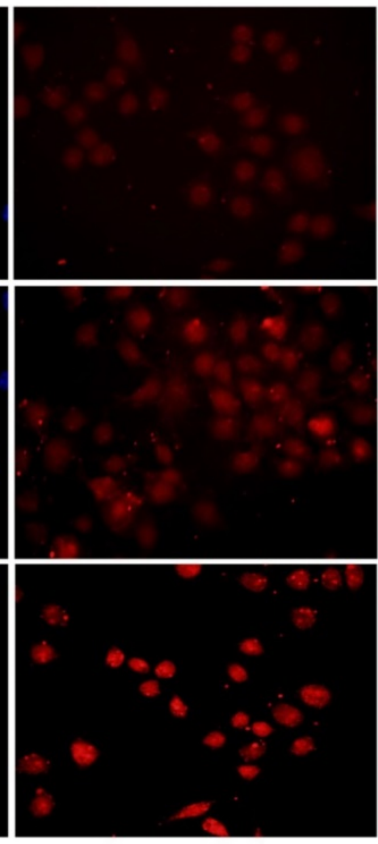

MERGE

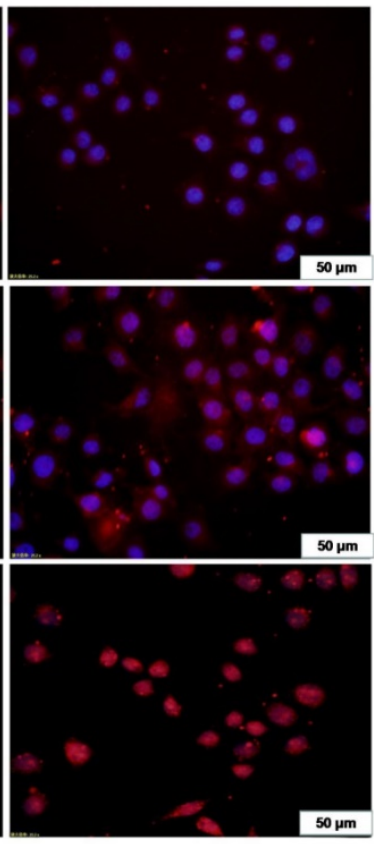

Fig 4. Targetted nanoparticles in HeLa cells observed by confocal microscope at different time interval. (A), (B) and (C) were determined after 1,2 , $4 \mathrm{~h}$ treatment, respectively. As the time of drug action increased, the fluorescence intensity was also increasing. When the cells were treated for $1 \mathrm{~h}$, the fluorescence intensity was significantly lower than $4 \mathrm{~h}$. The red fluorescence was not evenly distributed in the cells 4 hours later, but was partially aggregated, indicating that the drug was selective and not passive. The C-PC/CMC-CD59sp-NPs achieved targeted delivery to HeLa cells and the transportation was time-dependent. 


\section{Morphological changes observed under the confocal microscope}

The CD59sp was labeled by FITC, and then the C-PC/CMC-CD59sp-FITC NPs were prepared. Under confocal microscope, as shown in figure 5 , when HeLa cells were treated by targeted C-PC/CMC-CD59spFITC NPs for $48 \mathrm{~h}$, some cells showed irregular and polyangular. Most cells were still adherent growth but partial cells began to turn round. Surrounding cells began to bubble and apoptotic bodies formed. Cell contour was no longer clear, and the edge was irregular. Thus, under the actions of drugs, cells began to change their shape and apoptotic morphology appeared.

\section{The change of cell cycle detected by flow cytometry}

DNA contents of each phase of the cell cycle in four groups detected by flow cytometry were shown in figure 6, drugs could prevent HeLa cells from G1 phase to $S$ phase and $M$ phase, the inhibitory effects were more obvious in the targeted nano drug. So that the C - PC could cause G1 phase arrest of HeLa cells, and prevent DNA replication and cell division, while the effects of targeted drugs (C-PC/CMC-CD59sp) were more apparent.

\section{Targeted nanoparticles suppressed tumor growth in vivo}

Subsequently, we evaluated the impact of drugs on a HeLa xenograft model. Nude mice bearing subcutaneous HeLa cell xenografts were treated with either CMC-CD59sp, CMC/C-PC, CMC/C-PC-CD59sp or normal saline as a vehicle control $(n=5)$. None of the mice in the group with drugs treatment exhibited any weight loss or overt toxicity compared to the controls. All animals survived until the end-point of the study. Through 2-3 weeks of observation and measurement, we got the following growth curve, as shown in Fig. 7, the growth of HeLa xenografts was progressively suppressed in the CMC-CD59sp and CMC/C-PC treatment groups. The inhibitory effects of targeted nanoparticles were the most significant. After the mice were sacrificed, the weight and volume of tumors were measured. We found that the results were consistent with the results of tumor growth. The results showed that CMC-CD59sp-NPs and C-PC/CMC-NPs could inhibit tumor growth, but the inhibitory effects of C-PC/CMC-CD59sp-NPs particularly obvious.

\section{Bcl-2/ Cleaved caspase-3 protein level determined by immunofluorescence}

Bcl-2, known as an anti-apoptotic protein, prevents the release of cytochrome $\mathrm{C}$ from mitochondria to cytosol to inhibit cell apoptosis. As shown in figure $8 \mathrm{~A}$, compared with the control group, the $\mathrm{Bcl}-2$ expression quantities in other groups were significantly decreased, and the inhibitory effects of targeted nanoparticles was the most significant.

As shown in figure 8B, compared with control group, expression quantities of cleaved caspase-3 proteins were obviously increased in C-PC/CMC-CD59sp NPs group. The results showed that C-PC/CMC NPs and C-PC/CMC-CD59sp NPs could promote cleaved caspase-3 protein expression so as to induce the apoptosis of cells, while the targeted nanoparticles had more significant effects than other groups.

\section{Bcl-2/Cleaved caspase-3/Cyclin D1 protein expression detected by western blot}

Compare with control group, expression quantities of Bcl-2/ Cyclin D1 were apparently decreased in HeLa cells treated by CMC-CD59sp, C-PC/CMC NPs and C-PC/CMC-CD59sp, and the inhibitory effects of targeted nanoparticles were the most significant. Compared with control group, expression quantities of cleaved caspase-3 was significantly increased in CMC-CD59sp group and C-PC/CMC NPs group, while the C-PC/CMC-CD59sp NPs group obviously higher than that of the other two groups. So CMC-CD59sp, C-PC/CMC NPs and C-PC/CMC-CD59sp could promote cleaved caspase 3 protein expression but inhibit Bcl-2/ Cyclin D1 protein expression, further promote transduction of apoptotic signals. However the targeted nanoparticles had more significant effects than other groups (Figure 9).
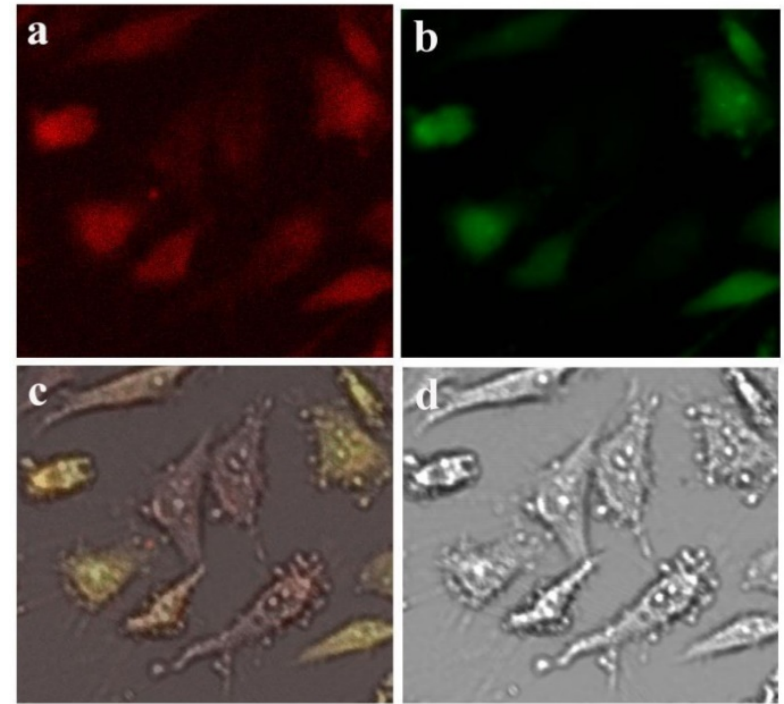

Fig 5. Apoptotic images of HeLa cells. (a) Cells treated by C-PC; (b) Cells treated by FITC labelled C-PC/CMC-CD59sp-FITC-NPs; (c) Merged figure; (d) White light. HeLa cells treated with C-PC/CMC-CD59sp-FITC-NPs for 48h, some cells showed irregular, polygonal and most still adherent, but partial individual cells begun to turn round, bubble and apoptotic bodies formed. The cell contour was no longer clear and the edge was not regular. 


\section{Discussion}

In recent years, the combination of nanotechnology and biomedicine promote research and development of drugs into a new direction. The size of the nanoparticles gives it advantage on drug delivery, changes the distribution and metabolism in organisms of traditional drugs, and make drug able to play the greatest roles. Some hidden lesions can emerge and get timely detection and treatment. At the same time, a variety of materials (nucleic acids, peptides, proteins, chemotherapy drugs, photosensitivity, fluorescent probe molecules) can be oriented to the target site and slowly released by targeted nanoparticles. The drug usage is maximized, but the side effects are minimized [17].

Chitosan, a kind of polysaccharides by deacetylation of chitin, is an abundant natural polymer, but isn't dissolved in water. O-CMC is a product of carboxylation of chitosan, and possesses good water solubility, so it is widely applied in cosmetics, pharmaceutical, especially acts as the carrier of nanoparticles. Drugs are stable in neutral aqueous solution by adsorption or parcel of O-CMC, and maintain pharmacological activity, delay the drug release and increase drug absorption time. Snima [18] prepared carboxymethyl chitosan nanoparticles entrapping metformin by ionic crosslinking, and cellular experiments proved obvious toxic effects of nano-drug on the pancreatic cancer cells. Maya [19] used $\mathrm{CaCl}_{2}$ as crosslinking agent to prepare targeting nanoparticles in which paclitaxel was embedded by O-CMC with EGFR as targets, and proved the targeting nanoparticels could realize the targeted therapy for tumors with high EGFR expression. Zhang [20] took LDL receptors as targets which were highly expressed on the surface of tumor cells by N-succinyl chitosan packaging osthole and then finished the targeted delivery and killing tumor cells.
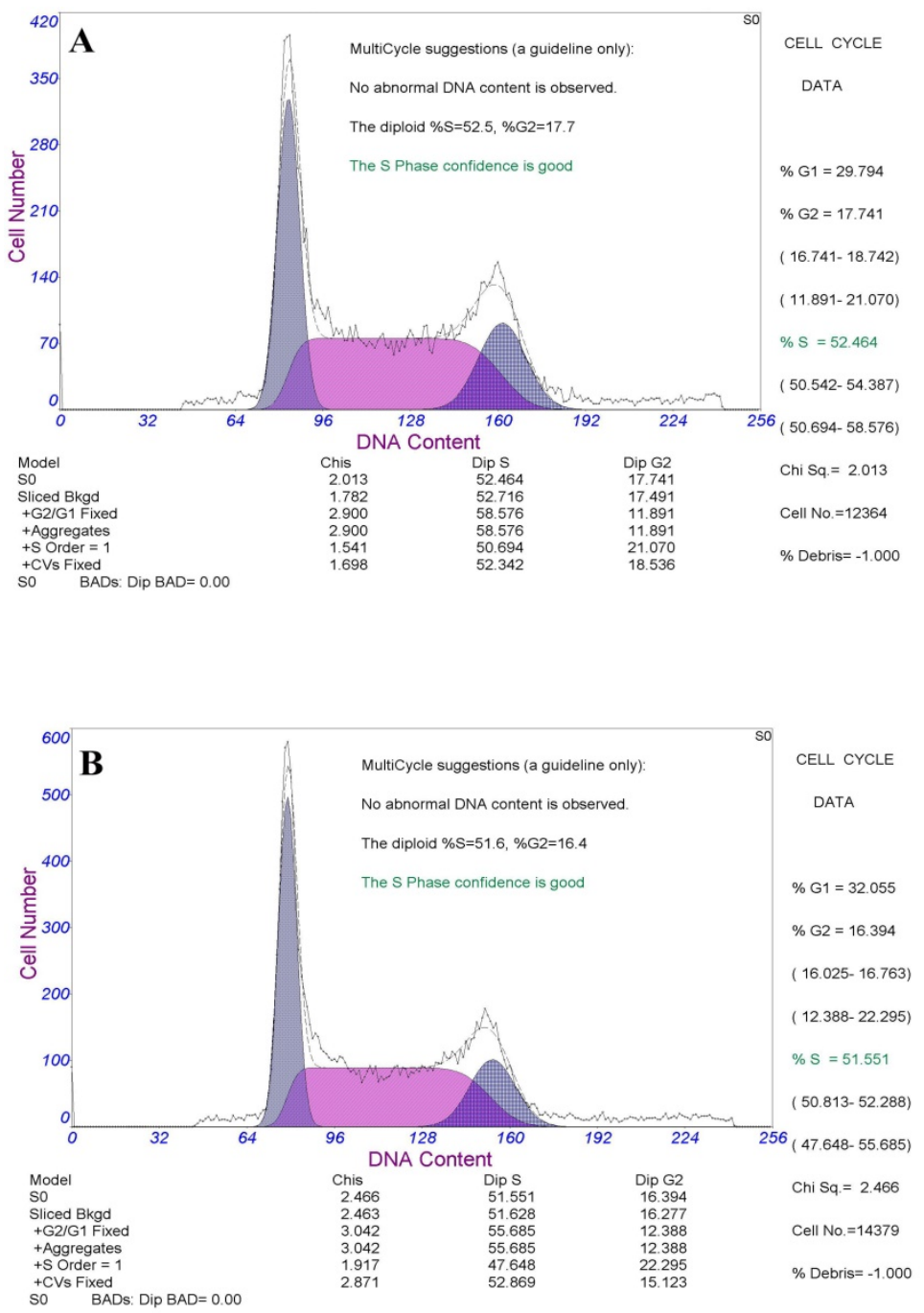

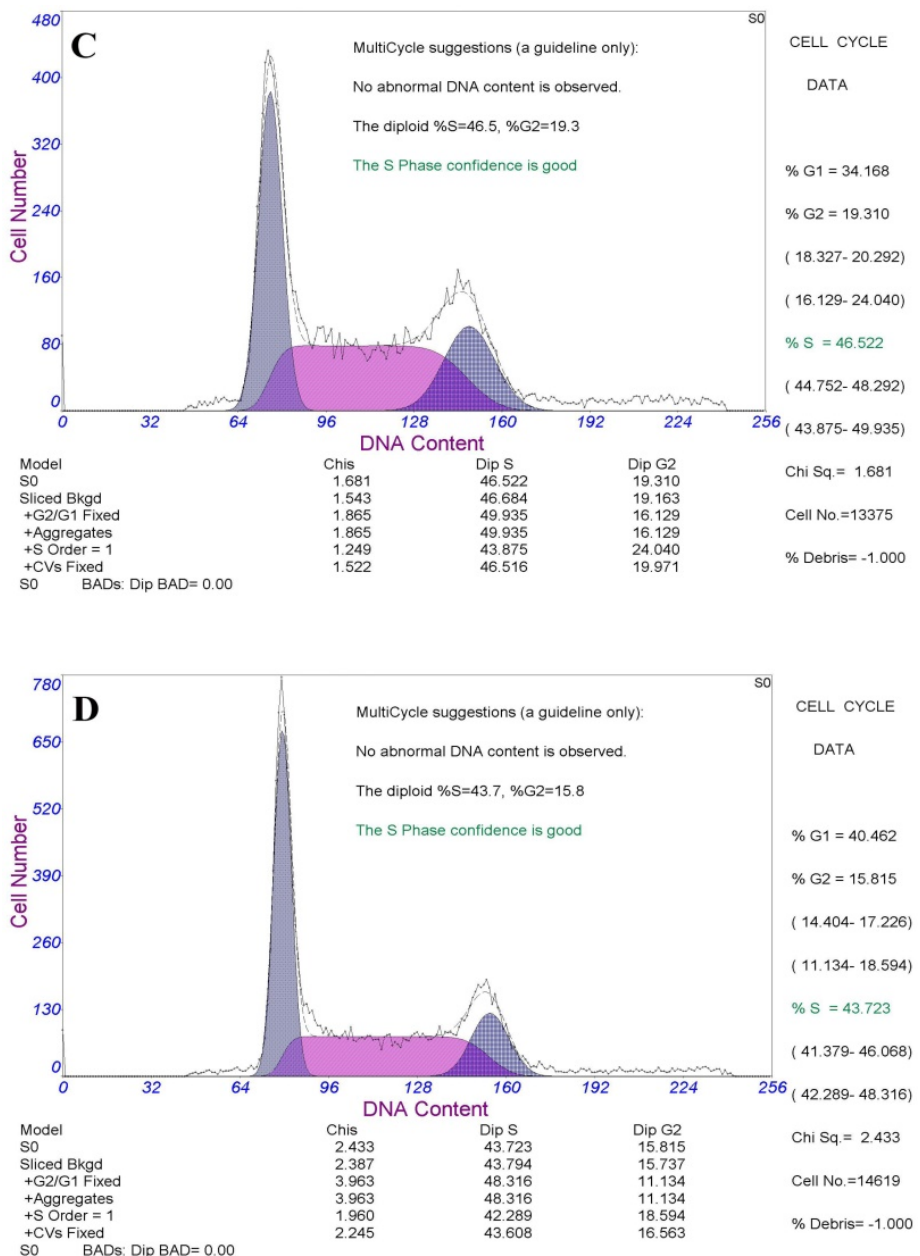

Fig 6. The changes of cell cycle detected by flow cytometry. (A) Control group. The proportion of G1 phase is $29.8 \%$. (B) C-PC treatment group. The proportion of G1 phase is $32.1 \%$. (C) C-PC/CMC treatment group. The proportion of G1 phase is $34.2 \%$. (D) C-PC/CMC-CD59sp treatment group. The proportion of Gl phase is $40.5 \%$. The C - PC could cause Gl phase arrest of HeLa cells, and prevent DNA replication and cell division, while the effects of targeted drugs (C-PC/CMC-CD59sp) were more apparent.

Our previous studies have found that C-PC has significant anti-tumor immune effects on a wide variety of tumor cells, such as ovarian cancer cells (SVOA) and breast cancer cells (MCF-7) cells [21]. However, the stability of C-PC is poor and it is easily degradable. CD59 molecule has high expression on the surface of plenty of solid tumors, so it can be used as the target spot of tumor therapy. In addition, the specific ligand peptide of CD59 (CD59sp) has been screened. So we proposed in combination with nano biomedical technology, taking C-PC as an anti-tumor drug, $\mathrm{CaCl}_{2}$ as crosslinking agent, $\mathrm{CMC}$ as embedding agent to prepare C-PC/CMC NPs by ionic crosslinking method. Taking advantage of EDC activation, CD59sp was connected to C-PC/CMC NPs to construct the targeted nanodrugs, which aimed at using specificity combination of CD59 peptide ligands and CD59 proteins on the surface of the tumor cells to fulfill the targeting intake of drug into cells under the guidance of CD59sp.

The experimental results showed that the morphology of the the nanoparticles finally prepared was well distributed with a diameter distribution of $200 \pm 11.3 \mathrm{~nm}$ and zeta potential of $-19.5 \pm 4.12 \mathrm{mV}$ measured by laser particle analyzer. MTT experiment results indicated that the drugs inhibited the proliferation of HeLa cells. IC50 value of targeted nanoparticels was $21.46 \mu \mathrm{g} / \mathrm{ml}$, and the inhibiting effect was more apparent than other groups, so that the C-PC activities in nanoparticles were well retained and the inhibitory efficacy to tumor cells was enhanced.

High expression of CD59 gene inhibited the formation of complement attack complex (MAC), which made the immune system lose the ability to precisely identify the tumor cells and caused immune escape [22]. On one hand, we made use of specific combination of CD59sp and CD59 molecule. CD59sp, as a targeted guide, could instruct the nanoparticles to achieve targeted drug delivery. On the other hand, CD59sp could seal active site of CD59, promote the formation of the MAC, and enhance anti-tumor 
effects. Compared with normal cells, HeLa cells absorbed more targeted nanoparticles within $1 \mathrm{~h}$, which was more than the intake amount of normal L929 cells within $4 \mathrm{~h}$ observed under the confocal microscope. At the same time, the intake of drugs was time-dependent. With the passage of time, more drugs selectively entered and gathered in cells.

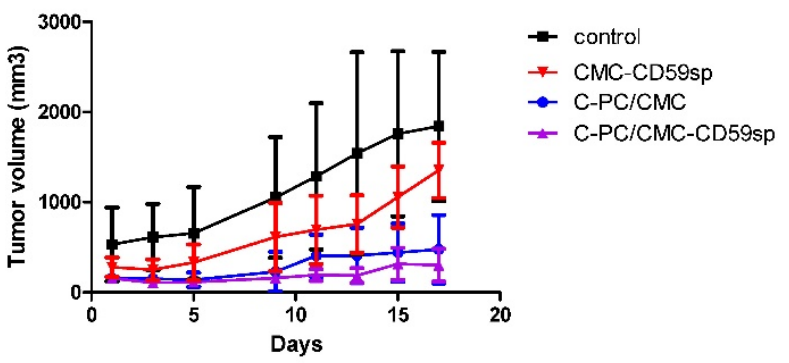

B

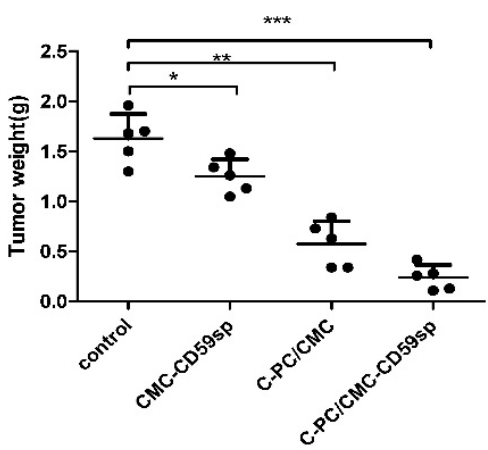

Fig 7. Targeted nanoparticles suppressed tumor growth in vivo. (A) Tumor volume. The growth of Hela xenografts was progressively suppressed in the CMC-CD59sp and CMC/C-PC treatment groups. The inhibitory effects of targeted nanoparticles were the most significant. (B) Tumor weights. *P $<0.05$ vs Ctrl group, $* * \mathrm{P}<0.01$ vs $\mathrm{C}$ trl group, ${ }^{*} * * \mathrm{P}<0.001$ vs $\mathrm{C}$ trl group.

A

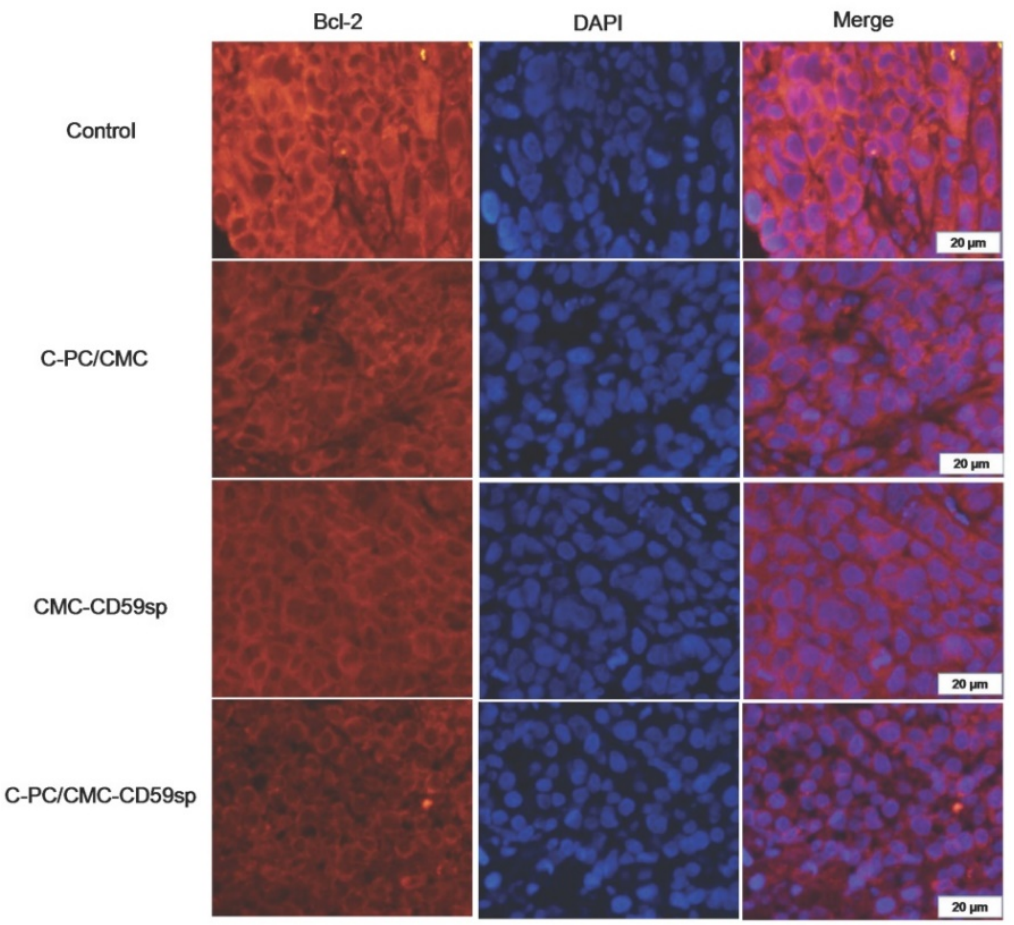


B

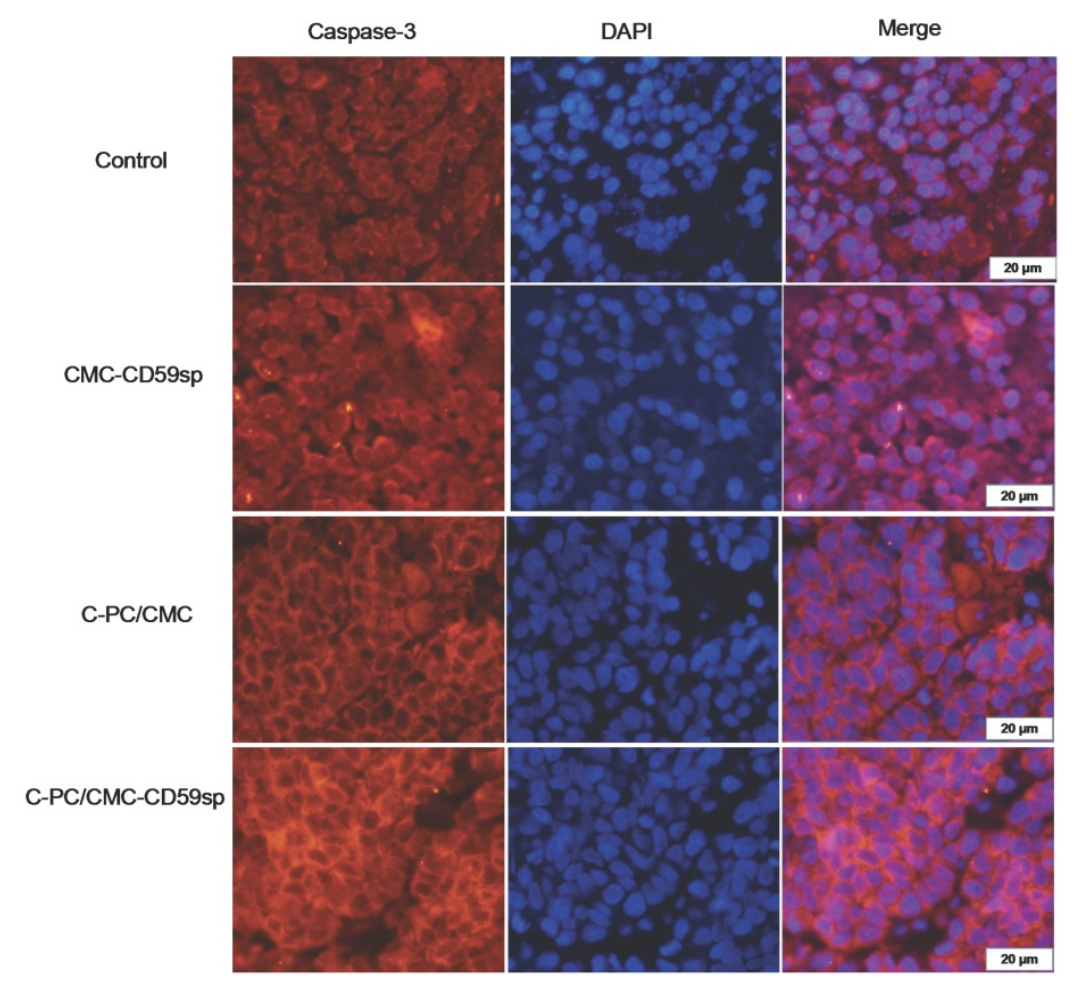

Fig 8. Bcl-2/ Cleaved caspase-3 protein level determined by immunofluorescence. (A) Immunofluorescent analysis of Bcl-2 in Hela cells. Compared with the control group, the expression of Bcl-2 in the CMC-CD59sp and C-PC/CMC-NPs groups was significantly lower than that in the control group, C-PC/CMC-CD59sp-NPs group was more obvious. (B) Immunofluorescent analysis of cleaved caspase-3 in Hela cells. Compared with the control group, the expression of the other groups was significantly increased, while the targeting of the nano-microspheres group was more obvious.

Abnormal proliferation of normal cells can lead to tumor. The cell division is out of control and cells lose their differentiation capacity, so continuously proliferate. Every cell divides once after one cell cycle, in which there is a restriction point $(R)$ during the transition process from $G 1$ phase to $S$ phase $[23,24]$. Because of the influence of internal and external factors, after G1 phase normal cells will develop to three directions: the proliferation, differentiation, and dormancy (G0). Tumor cells always proliferate and proliferation can't be limited. Our studies showed that C-PC/CMC-CD59 NPs could influence the HeLa cell cycle, causing G1 phase arrest, and cells cannot pass through $\mathrm{R}$ point to finish the cell proliferation, thus the proliferation of tumor cells was slowed down. Gene regulation also plays a very important role in the process of cell proliferation. Under normal circumstances, regulation to the restriction and promotion of proliferation is out of balance, but when an abnormity occurs, the proto-oncogenes are activated and tumor suppressor gene lose their original function, tumor cells form. So the next step we will in-depth study effects of the the nano targeted drugs on regulating the cell cycle and nuclear genes.

More and more studies have shown that cell cycle-related protein expression out of control on the occurrence and development of tumors play a direct role in promoting $[25,26]$. The molecular events controlling the G1 phase of cell cycle are determined by a series of phosphorylation events that are regulated by the expression of specific cyclins, cyclin-dependent kinases (cdks), and cdk inhibitors [27]. CyclinD1, which regulates progression through early phase of G1, exerts its effects by forming a holoenzyme complex with either CDK-4 or CDK-6. In our study, we observed the expression of CyclinD1 protein and results suggested that compared with control group, the level of CyclinD1 protein in CMC-CD59sp and C-PC/CMC group was obviously decreased, and the inhibitory effects of C-PC/CMC-CD59sp targeted nanoparticles were more significant. The results confirmed that C-PC/CMC-CD59sp targeted nanoparticles could induce more cell cycle arrest than control group by decreasing Cyclin D1 expression, and then inhibit the proliferation of HeLa cells.

The abnormal apoptosis of cells can also cause tumor. The apoptosis signal transduction is the key to cell apoptosis. Bcl-2 protein family and caspase family play a key role in cell apoptosis [28]. Bcl-2 proteins can inhibit cytochrome $\mathrm{C}$ release from mitochondria to cytosol, and then prevent the occurrence of 
apoptosis[29]. The apoptosis pathway induced by caspase protein family includes the exogenous and endogenous ways, but in the end cell apoptosis are caused by caspase- 3 activation. In general, Bcl-2 proteins lie in the upstream of caspase-3, and hold back caspase-3 activities. Our study detected apoptosis related proteins expression of HeLa cells before and after drug treatment. The results revealed that cleaved caspase- 3 protein expressions were promoted, but Bcl-2 protein expression quantities were lowered, and at the same time we found the targeted nanoparticles group had more obvious effects than other groups. Based on the above experimental results, we thought that the apoptosis of HeLa cells might be induced by different forms of the drugs, but the effects of C-PC/CMC-CD59sp targeted nanoparticles on tumor apoptosis were more significant. Increase of Bcl-2 proteins, decrease of caspase- 3 proteins expression and the formation of apoptotic bodies indicated the appearance of HeLa cell apoptosis after drug treatment. The occurrence of apoptosis might be the outcome of combined action of multiple pathways, so we need to further study other pathways in the future.

Therefore, targeted C-PC/CMC-CD59 nanoparticles induced apoptosis of HeLa cells might be fulfilled by the following steps: firstly, C-PC/CMC-CD59 nanoparticles were guided to reach the surface of HeLa cells by specific combination of CD59sp with CD59 molecules, and then by receptor mediated endocytosis, the drug was taken into cells. Subsequently, O-CMC was degraded, C-PC was gradually released, thereby inhibited the expression of Bcl-2 proteins and triggered a lot of cytochrome $\mathrm{C}$ released into the cytoplasm, which in turn activated caspase family such as caspase-3, finally induced the cells apoptosis and the formation of apoptotic bodies.

A

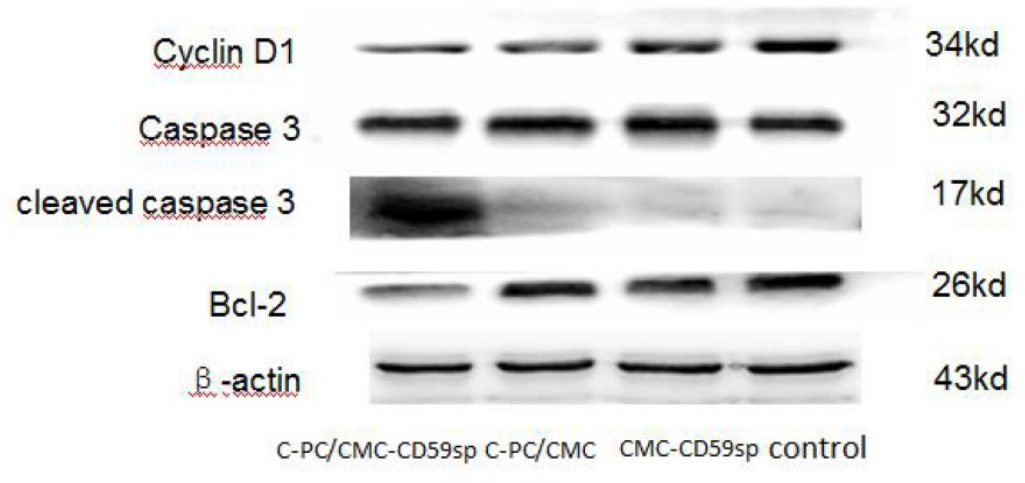

B

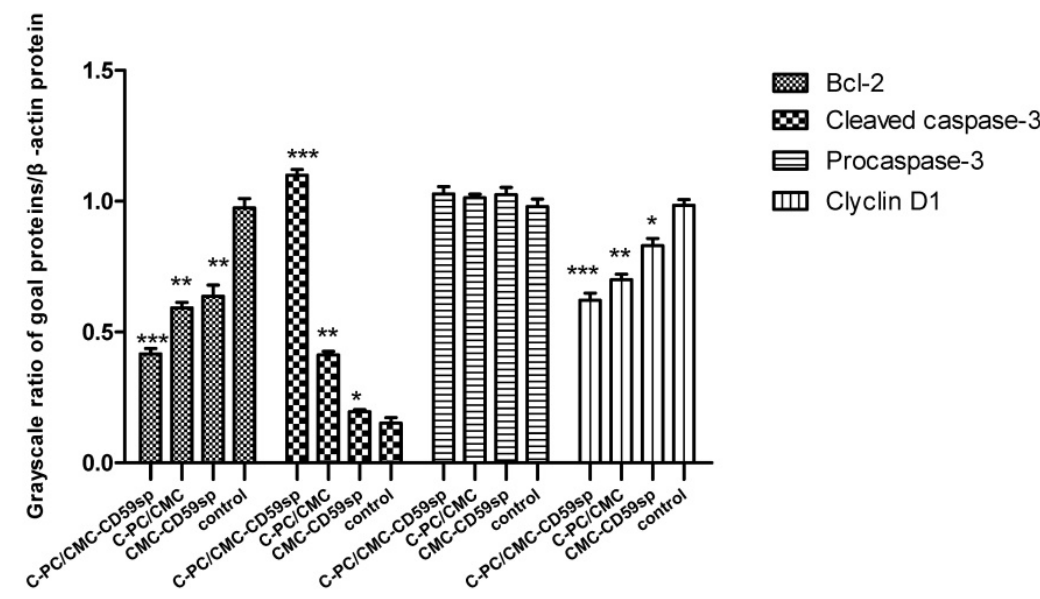

Fig 9. Detection of cleaved caspase-3/Bcl-2/Cyclin DI quantities in HeLa cells by Western blot. Compared with the control group, the expression of cleaved caspase-3 was significantly increased in experimental groups. Meanwhile, cleaved caspase-3 expression quantities in target NPs group were obviously higher than other groups. However, procaspase-3 proteins were of no significant changes in different groups. Compare with control group, expression quantities of Bcl-2/ Cyclin DI were apparently decreased in HeLa cells treated by CMC-CD59sp, C-PC/CMC NPs and C-PC/CMC-CD59sp, and the inhibitory effects of targeted nanoparticles were the most significant. (A) Electrophoretogram of target protein and $\beta$-actin in HeLa cells. (B) The grayscale ratio of target protein $/ \beta$-actin. $* \mathrm{P}<0.05$ vs control group, ${ }^{* *} \mathrm{P}<0.01$ vs control group, ${ }^{* * *} \mathrm{P}<0.001$ vs control group. 


\section{Abbreviations}

C-PC, C-phycocyanin; CMC, Carboxymethyl chitosan; NPs, nanoparticle, CD59sp, CD59- specific ligand peptide.

\section{Acknowledgments}

This work was supported by grants from the National Natural Science Foundation of China (81471546, 81001346, 81273206).

\section{Competing Interests}

The authors have declared that no competing interest exists.

\section{References}

1. Masood F. Polymeric nanoparticles for targeted drug delivery system for cancer therapy. Materials Science \& Engineering C. 2016; 60: 569.

2. Kuddus $M$, Singh P, Thomas G, Al-Hazimi A. Recent developments in production and biotechnological applications of C-phycocyanin. Biomed Res Int. 2013; 2013: 742859.

3. Pleonsil P, Soogarun S, Suwanwong Y. Anti-oxidant activity of holo- and apo-c-phycocyanin and their protective effects on human erythrocytes. International Journal of Biological Macromolecules. 2013; 60: 393-8.

4. Kim JA, Kim SK. Bioactive Peptides from Marine Sources as Potential Anti-Inflammatory Therapeutics. Current Protein \& Peptide Science. 2013; 14: $177-82$

5. Li B, Gao MH, Zhang XC, Chu XM. Molecular immune mechanism of C-phycocyanin from Spirulina platensis induces apoptosis in HeLa cells in vitro. Biotechnology and Applied Biochemistry. 2006; 43: 155-64.

6. Yang F, Li B, Chu XM, Lv CY, Xu YJ, Yang P. Molecular mechanism of inhibitory effects of C-phycocyanin combined with all-trans-retinoic acid on the growth of HeLa cells in vitro. Tumor Biology. 2014; 35: 5619-28.

7. Narayanan D, Jayakumar R, Chennazhi KP. Versatile carboxymethyl chitin and chitosan nanomaterials: a review. Wiley Interdisciplinary Reviews-Nanomedicine and Nanobiotechnology. 2014; 6: 574-98.

8. Wang YW, Qin F, Tan H, Zhang Y, Jiang M, Lu M, et al. pH-responsive glycol chitosan-cross-linked carboxymethyl-beta-cyclodextrin nanoparticles for controlled release of anticancer drugs. International Journal of Nanomedicine. 2015; 10: 7359-70.

9. Lubambo AF, Ono L, Drago V, Mattoso N, Varalda J, Sierakowski MR, et al. Tuning $\mathrm{Fe} 3 \mathrm{O} 4$ nanoparticle dispersion through $\mathrm{pH}$ in PVA/guar gum/electrospun membranes. Carbohydrate Polymers. 2015; 134: 775-83.

10. Bao HM, Zhang Q, Xu H, Yan Z. Effects of nanoparticle size on antitumor activity of 10-hydroxycamptothecin-conjugated gold nanoparticles: in vitro and in vivo studies. International Journal of Nanomedicine. 2016; 11: 929-40.

11. Sabuncu S, Culha M. Temperature-dependent breakdown of hydrogen peroxide-treated $\mathrm{ZnO}$ and $\mathrm{TiO} 2$ nanoparticle agglomerates. Beilstein Journal of Nanotechnology. 2015; 6: 1897-903.

12. Lu YY, Fan Y. Combined action of EGFR tyrosine kinase inhibitors and whole-brain radiotherapy on EGFR-mutated non-small-cell lung cancer patients with brain metastasis. Oncotargets and Therapy. 2016; 9: 1135-43.

13. Zhu DT, Chen C, Purwanti YI, Du SH, Lam DH, Wu CX, et al. Induced Pluripotent Stem Cell-Derived Neural Stem Cells Transduced with Baculovirus Encoding CD40 Ligand for Immunogene Therapy in Mouse Models of Breast Cancer. Human Gene Therapy. 2014; 25: 747-58.

14. Sivasankar B, Longhi MP, Gallagher KME, Betts GJ, Morgan BP, Godkin AJ, et al. CD59 Blockade Enhances Antigen-Specific CD4(+) T Cell Responses in Humans: A New Target for Cancer Immunotherapy? Journal of Immunology. 2009; 182: 5203-7.

15. Li B, Gao MH, Chu XM, Xu YJ, Yang F. Identification of a novel short peptide seal specific to CD59 and its effect on HeLa cell growth and apoptosis. Cellular Oncology. 2012; 35: 355-65.

16. Couvreur P, Barratt G, Fattal E, Legrand P, Vauthier C. Nanocapsule technology: A review. Critical Reviews in Therapeutic Drug Carrier Systems. 2002; 19: 99-134.

17. Korsmeyer R. Critical questions in development of targeted nanoparticle therapeutics. Regenerative Biomaterials. 2016; 3: 143-7.

18. Snima KS, Jayakumar R, Unnikrishnan AG, Nair SV, Lakshmanan VK. O-Carboxymethyl chitosan nanoparticles for metformin delivery to pancreatic cancer cells. Carbohydrate Polymers. 2012; 89: 1003-7.

19. Maya S, Kumar LG, Sarmento B, Rejinold NS, Menon D, Nair SV, et al. Cetuximab conjugated O-carboxymethyl chitosan nanoparticles for targeting EGFR overexpressing cancer cells. Carbohydrate Polymers. 2013; 93: 661-9.

20. Zhang CG, Zhu QL, Zhou Y, Liu Y, Chen WL, Yuan ZQ, et al. N-Succinyl-chitosan nanoparticles coupled with low-density lipoprotein for targeted osthole-loaded delivery to low-density lipoprotein receptor-rich tumors. International Journal of Nanomedicine. 2014; 9: 2919-32.

21. Li B, Gao MH, Chu XM, Teng L, Lv CY, Yang P, et al. The synergistic antitumor effects of all-trans retinoic acid and C-phycocyanin on the lung cancer A549 cells in vitro and in vivo. European Journal of Pharmacology. 2015; 749: 107-14.

22. Kesselring R, Thiel A, Pries R, Fichtner-Feigl S, Brunner S, Seidel P, et al. The complement receptors CD46, CD55 and CD59 are regulated by the tumour microenvironment of head and neck cancer to facilitate escape of complement attack. European Journal of Cancer. 2014; 50: 2152-61.

23. Tenga MJ, Lazar IM. Proteomic snapshot of breast cancer cell cycle: G1/S transition point. Proteomics. 2013; 13: 48-60.

24. Zhao XX, Chang JJ, Wang QL, Lu R, Li LJ, Sun $X$, et al. 5,6-Dihydroxy-3,7,4'-trimethoxyflavonol induces G2/M cell cycle arrest and apoptosis in human hepatocellular carcinoma cells. J Asian Nat Prod Res. 2016; 18: 1079-90.

25. Gautschi O, Ratschiller D, Gugger M, Betticher DC, Heighway J. Cyclin D1 in non-small cell lung cancer: a key driver of malignant transformation. Lung Cancer. 2007; 55: 1-14.

26. Jackson KM, DeLeon M, Verret CR, Harris WB. Dibenzoylmethane induces cell cycle deregulation in human prostate cancer cells. Cancer Lett. 2002; 178: 161-5.

27. Yu B, Lane ME, Pestell RG, Albanese C, Wadler S. Downregulation of cyclin D1 alters cdk 4- and cdk 2-specific phosphorylation of retinoblastoma protein. Mol Cell Biol Res Commun. 2000; 3: 352-9.

28. Saber A, Alipour B, Faghfoori Z, Mousavi Jam A, Yari Khosroushahi A. Secretion metabolites of probiotic yeast, Pichia kudriavzevii AS-12, induces apoptosis pathways in human colorectal cancer cell lines. Nutr Res. 2017.

29. Yuan ZF, Tang YM, Xu XJ, Li SS, Zhang JY. 10-Hydroxycamptothecin induces apoptosis in human neuroblastoma SMS-KCNR cells through p53, cytochrome c and caspase 3 pathways. Neoplasma. 2016; 63: 72-9. 